\title{
INFORMAÇÕES TÉCNICAS PARA SELEÇÃO DE CAVALOS MECÂNICOS PARA O TRANSPORTE RODOVIÁRIO
}

\author{
Bruno Bortoluzzi Benetti $^{1}$, Catize Brandelero ${ }^{2}$, Eric Porto Gindri ${ }^{1}$, Guilherme Magro da Silva ${ }^{1}$ Valmir Werner ${ }^{2}$ \\ 1 Curso de Engenharia Florestal, Universidade Federal de Santa Maria, 97105-900, Santa Maria, Brasil. \\ 2 Departamento de Engenharia Rural, Universidade Federal de Santa Maria, 97105-900, Santa Maria, Brasil.
}

E-mail:Llaboratoriomecaniza@gmail.com

\section{RESUMO}

Em virtude da quantidade de variáveis importantes existentes, este trabalho objetivou sintetizar informações técnicas que auxiliem na seleção dos cavalos mecânicos, como: motor (marca, modelo e potência), capacidade máxima de tração e volume dos tanques de combustível. A pesquisa foi elaborada com base em dados coletados nos catálogos dos modelos de cavalos mecânicos, disponíveis no mercado brasileiro, no ano de 2016. Os dados obtidos pela consulta aos catálogos foram tabulados em planilha eletrônica para efetuar a análise. No levantamento, foram identificados 135 modelos, disponíveis para comercialização neste ano. Com os resultados, observouse que os fabricantes de cavalos mecânicos predominantes no mercado brasileiro, pela quantidade de modelos ofertados, são da marca Scania, seguida por Volvo, Mercedes, Iveco e Volkswagen. Para os fatores de potência, capacidade máxima de tração e capacidade de tanque, a maior parte dos cavalos mecânicos produzidos no país busca atender os limites máximos de peso de carga transportado, determinados pela lei, estando nas Classes II e III estabelecidas no estudo, compostas por valores medianos.

Palavras-chave: Unidades tratoras. Transporte rodoviário. Modelos.

\section{Introdução}

O transporte rodoviário é o modal mais utilizado no Brasil. Segundo CFA [1], este módulo de transporte é utilizado em $60 \%$ de tudo o que é transportado no Brasil. Conforme ANTT [2], no país, a frota rodoviária é composta por $1.951 .455,0$ veículos, com idade média de uso de 13 anos, divididos entre autônomos, empresas transportadoras e cooperativas.

De acordo com Koerich [3], o transporte rodoviário brasileiro obteve consolidação ao final da década de 50 e foi associado à implantação da indústria automobilística no país e a mudança da capital federal para a região Centro-Oeste. Tais modificações foram acompanhadas de um massivo programa de construção de rodovias por toda a região Sudeste e Centro-Oeste, principalmente. Padrão que se mantém atualmente, por falta de políticas de incentivo aos outros métodos de transporte, os quais o país tem potencial.
O Ministério dos Transportes cita que esse modal possui uma baixa capacidade de carga, com limitação de volume e peso. Assim, é necessária a utilização de diferentes tipos de veículos com capacidades de carga apropriadas. De acordo com o Ministério dos Transportes [4], os principais e mais utilizados são os caminhões e cavalos mecânicos com suas unidades de carga.

A ABNT [5] define o veículo rodoviário de carga como sendo todo o veículo utilizado para o trânsito nas vias de rolamento, destinado ao transporte geral de cargas. Dentro desta definição está o cavalo mecânico (Figura 1), que é um veículo automotor, equipado com quinta-roda, destinado a tracionar um implemento rodoviário. Segundo Machado et al. [6], o cavalo mecânico, combinado com unidades de carga, compõe os tipos de veículos conhecidos como: carreta, bitrem, tritrem e rodotrem. 

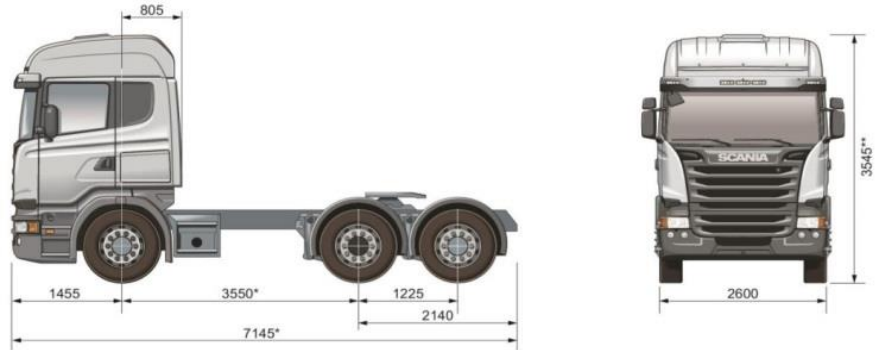

Figura 1 - Desenho demonstrativo de um cavalo mecânico. [7]

De acordo com Ministério dos Transportes [8], para a distribuição de massa por eixos isolados, de dois e quatro pneus, o limite máximo é de 6,0 T e 10,0 T, respectivamente. Já com dois e três eixos em tandem, de quatro pneus cada, o limite passa para $17,0 \mathrm{~T}$ e $25,5 \mathrm{~T}$, respectivamente. Os eixos isolados possuem uma distância entre eixos maior que 2,40 metros, conferindo um limite máximo maior e uma melhor distribuição do peso ao longo do veículo. Os eixos em tandem possuem uma distância entre eixo que varia de 1,35 a 2,40 metros, sendo o limite máximo diretamente proporcional a essa distância.

Conforme o CONTRAN [9], as combinações veiculares com mais de duas unidades não podem possuir PBTC (peso bruto total combinado) superior a 74,0 T, comprimento superior a 30 metros e o limite máximo por eixo deve ser respeitado. Esse limite é definido com base na potência do motor, sendo estabelecido de 4,2 kW/t (5,71 cv/t), restringindo-se, ainda, ao limite máximo de 45 t. Veículos com PBTC maior do que 45,0 T e até 57,0 T, com no máximo 7 eixos e comprimento máximo de 19,8 metros, podem trafegar sem a necessidade da autorização especial de trânsito (AET).

A escolha do veículo correto para o transporte é essencial à adequação ao trabalho, diminuindo a chance de acidentes e a necessidade frequente de manutenção. Características como composição física, geometria, peso, classificação, configuração dos eixos e tecnologias utilizadas são elementos essenciais para seleção do veículo na composição do transporte rodoviário de cargas [10].

Seixas [11] argumenta que, ao envolver longas distâncias e o tráfego em estradas de terra e asfalto, o transporte rodoviário exige o emprego de veículos pesados e com maior capacidade de carga. Considera-se também que o número de eixos na composição e o peso em cada eixo são fatores relevantes na seleção de veículos para os transportadores de cargas, visto que buscam otimizar a quantidade de produto transportado por veículo, contudo, sem ultrapassar os limites estabelecidos na legislação [12].
Diante da importância deste modal de transporte no país, e da gama de cavalos mecânicos ofertados no mercado com diferentes características, este trabalho objetiva estabelecer fatores para realizar a seleção destes veículos, em função de algumas características mecânicas como: motor (marca, modelo, potência e torque), capacidade máxima de tração e o volume dos tanques de combustível, em 135 modelos, comercializados no Brasil, no ano de 2016.

\section{Parte Experimental ou Metodologia}

A coleta dos dados foi realizada utilizando os catálogos técnicos disponibilizados pelas empresas fornecedoras de cavalos mecânicos, no Brasil, no ano de 2016. Os dados coletados foram: motor (marca, modelo, potência e torque), volume do tanque de combustível e capacidade máxima de tração (CMT); estes foram tabulados em planilha eletrônica.

Os cavalos mecânicos encontrados no levantamento foram organizados em 3 grupos, sendo cada um destes subdividido em quatro classes, conforme apresentado no Quadro 01. Os limites inferiores e superiores de cada classe, dentro dos diferentes grupos, foram criados pelos autores, após processamento dos dados contidos na planilha.

Quadro 01 - Classificação dos cavalos mecânicos em grupos, quanto a potência do motor, capacidade volumétrica dos tanques de combustível e capacidade máxima de tração.

\begin{tabular}{ccc}
\hline Grupos & Classes & $\begin{array}{c}\text { Limite inferior e } \\
\text { superior }\end{array}$ \\
\hline Potência do motor (kW) & I & 115,0 a 225,0 \\
& II & 226,0 a 336,0 \\
& III & 337,0 a 447,0 \\
CMT (T) & IV & 448,0 a 560,0 \\
Capacidade máxima de tração - & II & 38,0 a 66,0 \\
CMT, & III & 94,1 a 122,0 \\
Capacidade volumétrica de & IV & 122,1 a 150,0 \\
tanques de combustível (L) & III & 150,0 a 495,0 \\
& IV & $1.186,0$ a $1.530,0$ \\
\hline
\end{tabular}

TECNO-LÓGICA, Santa Cruz do Sul, v. 22, n. 1, p. 85-89, jan./jun. 2018. 


\section{Resultados e discussões}

No levantamento, foram computados 135 modelos comercializados por 10 marcas comerciais, como pode ser observado na Figura 2. A marca Scania foi a de maior representatividade no mercado brasileiro, com 64 modelos de cavalos mecânicos, totalizando $47,4 \%$ dos modelos comercializados no país. As marcas Iveco, Mercedes e Volvo possuem 13 modelos cada, tendo uma representatividade de 9,6\% do total, respectivamente, seguidos pela marca Volkswagen com 11 modelos, representando $8,1 \%$. A alta representatividade destas marcas pode ser explicada pelo histórico das mesmas, pela qualidade de seus produtos e por estarem presentes no Brasil, há vários anos. Os fabricantes com menor disponibilidade de modelos no mercado foram Shacman, Ford, Daf, Sinotruk e Man. Estes representaram, respectivamente, $4,4 \%, 3,7 \%, 3,0 \%, 2,2 \%$ e $2,2 \%$, dos modelos comercializadas no país.

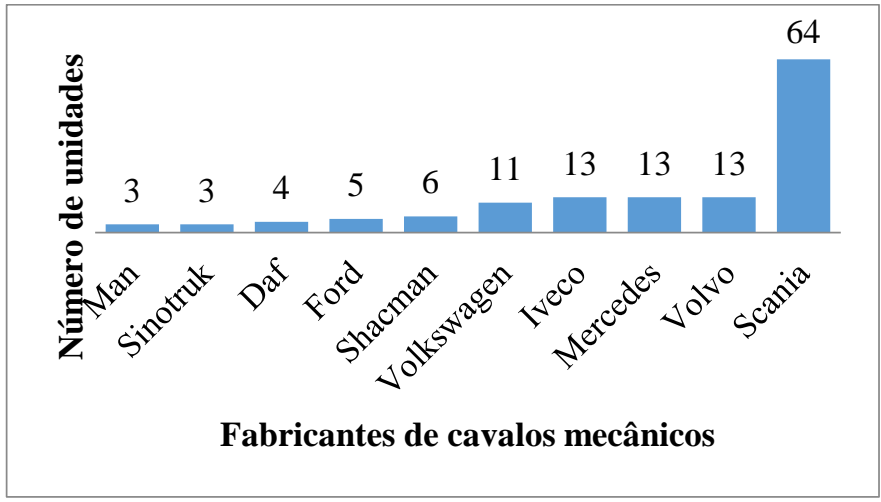

Figura 2 - Distribuição do número de cavalos mecânicos, comercializados por marca, no mercado brasileiro.

Com relação à potência dos motores nos cavalos mecânicos, 3,0\% foram enquadrados na Classe I; 65,9\% na Classe II; 25,2\% na Classe III dos modelos e 5,9\% na Classe IV (Figura 3 ). Pode-se explicar a maior concentração dos motores dos cavalos mecânicos na Classe II por estes modelos serem de potência média de, aproximadamente, $321,3 \mathrm{~kW}$. As classes I e IV de motores contêm potências afastadas da média, sendo constituídas por cavalos mecânicos que desempenham atividades especiais como autoescola na Classe I e, na Classe IV, transporte de equipamentos pesados, como geradores e turbinas.

Ao analisar as marcas de motores presentes nos cavalos mecânicos disponíveis no mercado, foram encontrados 8 fabricantes, distribuídos de acordo com a Figura 4.
As marcas de motores com maior predomínio nos cavalos mecânicos foram da Scania, com 64 modelos e Cummins com 20 modelos, seguidos da FPT e Mercedes, com 15 e 13 modelos, respectivamente.

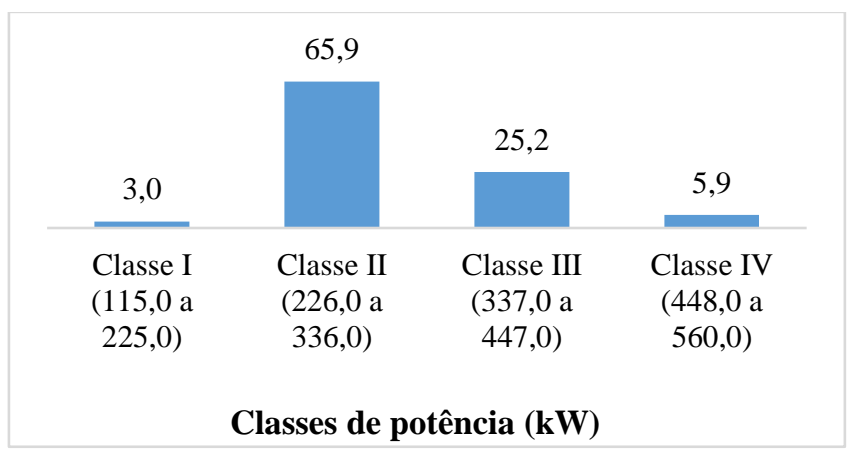

Figura 3 - Distribuição dos cavalos mecânicos quanto à classe de potência dos motores.

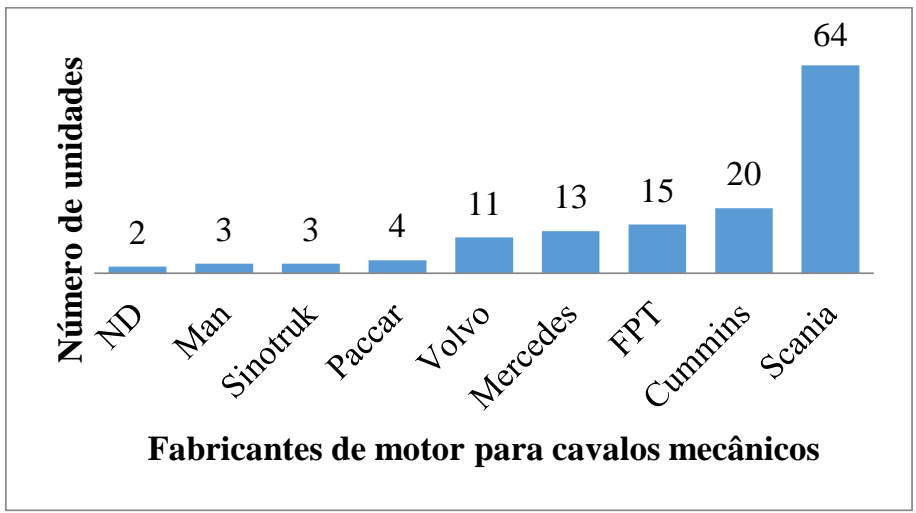

Figura 4 - Distribuição do número de modelos de motores por marcas disponíveis nos cavalos mecânicos.

O expressivo número de modelos de motores da marca Scania ocorre pela representatividade no mercado brasileiro e por possuírem fabricação própria. Na sequência, o motor Cummins se mostra representativo no mercado, sendo utilizado por marcas como: Ford, Shacman e Volkswagen.

Em relação às classes de tração, quase a totalidade dos cavalos mecânicos foram enquadrados nas Classes I e II, com porcentagem de $37,0 \%$ e $51,9 \%$, respectivamente. As Classes restantes, III e IV, representaram $1,5 \%$ e $7,4 \%$, respectivamente. Ainda, foram encontrados catálogos que não disponibilizaram o CMT dos modelos de cavalos mecânicos, totalizando $2,2 \%$ dos veículos. 
Segundo Machado et al [6], o PBTC para rodotrens e treminhões, deve ser de no máximo 74,0 T. Desse modo, é vista a grande representatividade de cavalos mecânicos das classes I (38,0 a 66,0 T) e II (66,1 a 94,0 T), pois com o limite imposto de 74,0 T é evitado, a compra de modelos com maior CMT para fins rodoviários. Assim essas duas classes compõem grande parcela do mercado, de cavalos mecânicos, brasileiro.

$\mathrm{Na}$ Figura 5, é possível observar a relação entre a capacidade de tanque de combustíveis dos cavalos mecânicos e as classes de potência.

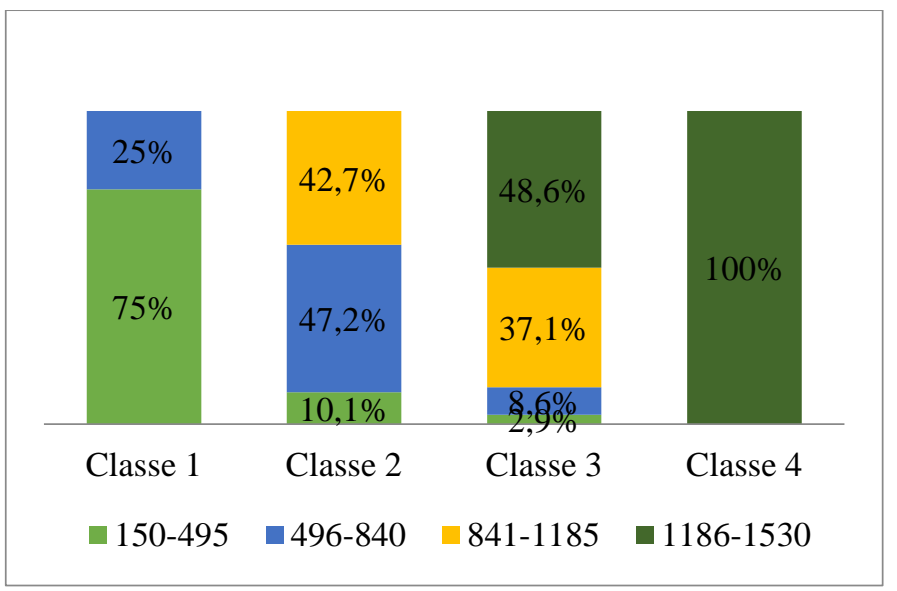

Figura 5 - Relação da capacidade de tanque dos cavalos-mecânicos com as classes de potência. (Fonte: Autores).

Ao analisar a relação da potência do motor com a capacidade de tanque, constatou-se que na Classe I de potência do motor, o predomínio é da Classe I de capacidade de tanque, representando o total de $75 \%$. Na Classe II de potência do motor, $42,7 \%$ e $47,2 \%$ dispõem da classe III e IV de capacidades de tanque, respectivamente. Na classe III de potência do motor, $48,6 \%, 37,1 \%, 8,6 \%$ e $2,9 \%$ são compostos pelas classes IV, III, II, I de capacidade de tanque, respectivamente. Já, a totalidade da Classe IV de potência do motor, foi constituída pela classe IV de capacidade de tanque.

É possível inferir que o aumento da capacidade de tanque dos cavalos-mecânicos é diretamente proporcional ao aumento da sua potência. Além disso, esse aumento de capacidade permite potencializar a eficiência operacional da máquina, reduzindo a necessidade de paradas para reabastecimento, possibilitando percorrer trajetos que compreendam uma maior distância, bem como realizar o abastecimento em postos que apresentem a qualidade desejada.

\section{Conclusões}

A escolha correta do cavalo mecânico é essencial, uma vez que está diretamente ligado à redução de custos, atrelados ao transporte rodoviário de cargas. Assim, ao dispor das informações técnicas necessárias dos veículos, o comprador terá maior facilidade no momento da tomada de decisão acerca da escolha do veículo e do uso adequado do mesmo.

De modo geral, seguindo as especificações técnicas, os cavalos mecânicos predominantes no mercado brasileiro, pertencem à marca Scania, sendo essa, a fabricante de seus motores. É uma característica importante na escolha do veículo.

Seguindo as características de potência, capacidade máxima de tração e capacidade de tanque, pode-se considerar que a maioria das marcas de cavalos mecânicos produzidos no país atendem os limites máximos de peso de carga transportado, impostos pela lei vigente. Constatou-se a predominância dos cavalos mecânicos nas classes intermediárias II e III estabelecidas.

\section{Agradecimentos}

MECANIZA/UFSM.

\section{TECHNICAL INFORMATION FOR THE SELECTION OF TRUCK-TRACTORS FOR ROAD TRANSPORT}

\begin{abstract}
Due to the number of important variables, this work aimed to synthesize technical information to assist in the selection of truck-tractors, such as: motor (make, model and power), maximum traction capacity and volume of fuel tanks. The research was elaborated based on data collected in the catalogs of models of truck-tractors available in the Brazilian market, in the year 2016. The data obtained by consulting the catalogs were tabulated in a spreadsheet to perform the analysis. In the survey, 135 models were identified, available for commercialization this year. With the results, it was observed that the manufacturers of truck-tractors predominant in the Brazilian market, by the number of models offered, are of the Scania brand, followed by Volvo, Mercedes, Iveco and Volkswagen. For the characteristics of power, maximum traction capacity and tank capacity, most of trucktractors produced in the country seek to meet the maximum weight
\end{abstract}


limits of transported cargo established by law, being in Classes II and III established in the study, composed of medium values.

Keywords: Tractor units. Road transportation. Models.

\section{Referências}

[1] CFA - Conselho Federal de Administração, Plano Brasil de Infraestrutura e Logística. Disponível <http://www.cfa.org.br/servicos/publicacoes/planobrasil_web>;. Acesso em:

02 de Outubro de 2016.

[2] ANTT - Agência Nacional de Transportes Terrestres. <http://www.antt.gov.br/index.php/content/view/20271/Transportadores__Frota _Tipo_de_Veiculo.html>. Disponível em: 14 de Outubro de 2016.

[3] Koerich, A. et. Al; Transporte rodoviário de toras e toretes. Revista Campo \& Negócios - Florestas. n. 33. p. 44-46, 2017.

[4] Ministério dos Transportes - Transporte Rodoviário do Brasil. Disponível em: <http://www2.transportes.gov.br/bit/02-rodo/rodo.html>. Acesso em: 09 de Outubro de 2016.

[5] ABNT - ASSOCIAÇÃO BRASILEIRA DE NORMAS TÉCNICAS. Veículo rodoviário de carga - Terminologia. Brasil, 2006. Disponível em: <t;www.abnt.org.br>; Acesso em: 25 de Novembro 2017.

[6] Machado, C. C.; Machado, R. R.; Silva, A. E.; Gomes de Souza, M. F.; Avaliação do Desempenho Logístico do Transporte Rodoviário de Madeira de Áreas de Fomento Florestal Com o Uso de Rede de Petri [7] SCANIA - Manual de informações técnicas cavalo mecânico R 400 LA4x2 Highline c/3º eixo R885. Disponível em:

https://www.scania.com/content/dam/scanianoe/market/br/pdfs/especifica\%C3\% A7\%C3\%B5es/r/182_R400_LA4X2_Highline_com_3eixo_R885_tcm253469576.pdf>. Acesso em: 29 de Novembro de 2017.

[8] Ministério dos Transportes - Limites Legais. Disponivel em: <http://www1.dnit.gov.br/Pesagem/qfv\%20pdf.pdf>. Acesso em: 05 de Junho de 2017.

[9] CONTRAN - Conselho Nacional de Trânsito. Resolução 68/98- Requisitos de segurança necessários à circulação de Combinações de Veículos de Carga- CVC. Disponível em:

www.denatran.gov.br/download/Resolucoes/resolucao068_98.doc>. Acesso em: 25 de Novembro de 2017.

[10] Villela, T. M. A.; Tedesco, G. M. I. Sistema de transporte rodoviário de cargas: uma proposta para sua estrutura e elementos. Disponível: <https://www.revistatransportes.org.br/anpet/article/view/507/393>. Acesso em: 15 de Outubro de 2016.

[11] Seixas, F. Uma metodologia de seleção e dimensionamento da frota de veículos rodoviários para o transporte principal da madeira. São Carlos. 106 p. 1992. (Tese de Doutorado - EESC).

[12] Seixas, F. Novas Tecnologias no Transporte Rodoviário de Madeira. IPEF, Piracicaba. 2001. Disponível em:< http://www.ipef.br/silvicultura/transporte.pdf >; Acesso em: 24 de Novembro de 2017.

TECNO-LÓGICA, Santa Cruz do Sul, v. 22, n. 1, p. 85-89, jan./jun. 2018. 